\title{
Structure optimization of embedded scalable fire rescue equipment
}

\author{
Jianguo $\mathrm{LUO}^{1,}$, Zehao BU ${ }^{2, \mathrm{~b}}$ \\ ${ }^{1}$ Department of Mechanical and Electrical Engineering, North China Institute of Science and \\ Technology, East Suburb of Beijing, 101601, China \\ ${ }^{2}$ Department of Graduate School, North China Institute of Science and Technology, East Suburb of \\ Beijing, 101601, China

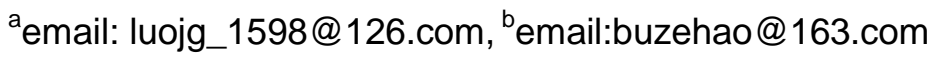

Keywords: High-rise Building Fire; Structural Analysis; ANSYS; Optimization Design

\begin{abstract}
Deeply analyzing the main performance parameters and best feature size of the outside telescopic arm of embedded retractable telescopic fire rescue equipment, according to the designed structure of the entire system, analyzing the structural performance parameters which affect its work so as to make optimizing analysis of it. Based on the ANSYS Workbench 15.0, make the structural analysis for static, fatigue resistance, susceptibility of the telescopic arm assembly model. Make comparing design according to the materials used for the design, cross-sectional shape of the pneumatic cylinder, the first stage cylinder, with Creo3.0 simulation do finite element analysis to make parameter optimization design so as to achieve the ultimate effect.
\end{abstract}

\section{Introduction}

High-rise building fire has become a tough problem for firefighting and rescue. In order to solve these problems, such as firefighting, evacuation, fire detection, making effective construction design, complemented by efficient mechanical system design and integrated design of modern surveillance and prevention has become the solution to the high-rise fire rescue [1]. The embedded scalable fire rescue equipment (Figure 1) is a comprehensive design for fire alarm, fire detection, extinguishing fire and personnel rescue. Before it is put into practice, it has to be made many improvements, with the modeling and simulation software, a variety of analysis is the most simple and most effective optimization method [2]. In order to achieve optimal performance, the overall structure of the finite element mechanical analysis based on ANSYS Workbench and Creo3.0 software [3], making analysis mainly for the material properties of parts, cross-sectional shape of the cylinder, the first stage cylinder parameters, during the analysis of the structure of parts, mainly for the size and quality of the parts [4]. In the condition of without influencing the actual use of the device, optimization process helps improve the device parameters, provide a theoretical basis for the optimization design, enhanced the reliability and usefulness of the device.

\section{Embedded retractable fire rescue equipment}

Pneumatic telescopic arm (Figure 2) is the most important part of the embedded scalable fire rescue equipment, with a large span cantilever structure, installed with fire extinguishing system and real-time monitoring equipment in it, which carry its load on telescopic arm, so there are strict requirements of it. The main part of the telescopic arm is multi-stage cylinders and the cylindrical metal parts which guide the linear motion actuator piston. Pneumatic cylinder is compressed by gas extrusion; gas expands under heating, turn the thermal energy into mechanical energy. According to the size of the force required under operating conditions, calculate the thrust and tensile force, the cylinder output capacity should be reserved to meet the security work. When judging the diameter, if the election is small, the output power is not enough, the cylinder cannot complete the work; if the diameter is too large, it will increase the cost of equipment, equipment is too heavy, air consumption increases, resulting in waste of energy. 


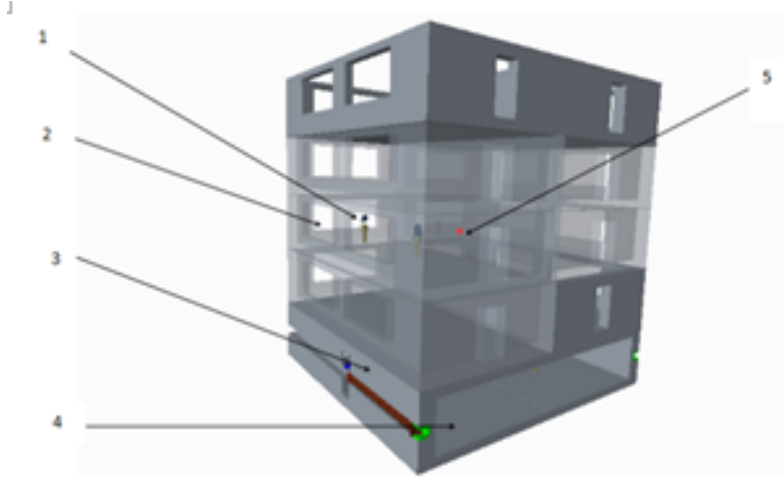

1. Human BodyModel 2 Fire floor 3. Embedded Scalable Systems. 4. Refuge floors 5.Fire source

Figure 1: 3D Models



Figure 2: Pneumatic telescopic system diagram

\section{Structure Analysis of ANSYS Workbench}

Modeling in PTC Creo3.0 Parametric, import the model into Workbench for analysis. After the modeling is finished, define the materials, loads and constraints of the model, after making a good division of the lattice structure, then move into the next step of the analysis. Here the main application of static analysis is to optimize the analysis which can get the parameters of stress, strain, and total displacement.

(1) Analyze what effect the cross-section of the cylinder will make for the entire institution, mainly analyze square and circular cross-sectional. Making static analysis performed and fatigue analysis according to cylinder section, static analysis results are shown in Figure 3, 4, 5.



Figure 3: Stress

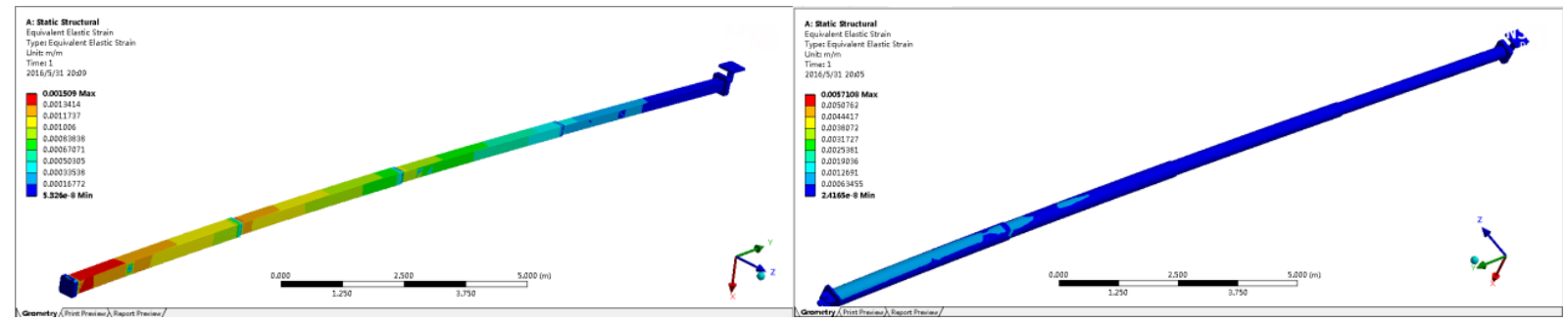

Figure 4: Strain

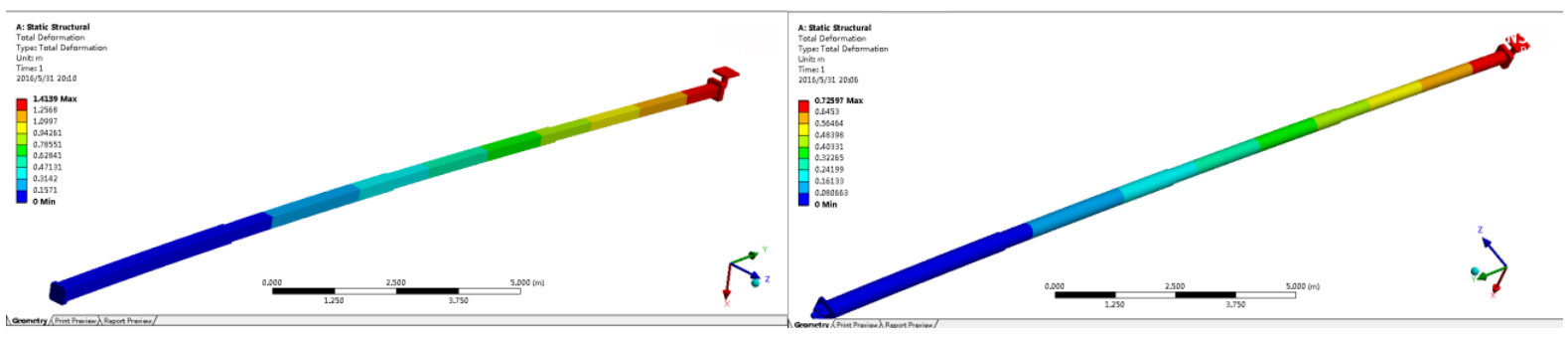

Figure 5: Total displacement

From cloud static analysis, we can see a circular cross section maximum stress is $5.16 \times 10^{8} \mathrm{~Pa}$, and square cross-section maximum stress is $2.24 \times 10^{8} \mathrm{~Pa}$ (Fig. 3); from the analysis of strain (Fig. 
4), the maximum strain of circle cross-section is $5.71 \times 10^{-3} \mathrm{~m} / \mathrm{m}$, square cross-section only is $2.41 \times 10^{-3} \mathrm{~m} / \mathrm{m}$, from the total displacement (Fig. 5), the square cross-section is only $0.49 \mathrm{~m}$, and a circular cross section is $0.73 \mathrm{~m}$. From the point of static analysis, square cross-section of the cylinder has more advantages.

Based on the above static analysis, making further comparative analysis of fatigue resistance, so as to get the relevant data of derived life, safety factors and the load - time curves, which is the main basis for comparison of performance across the organization.
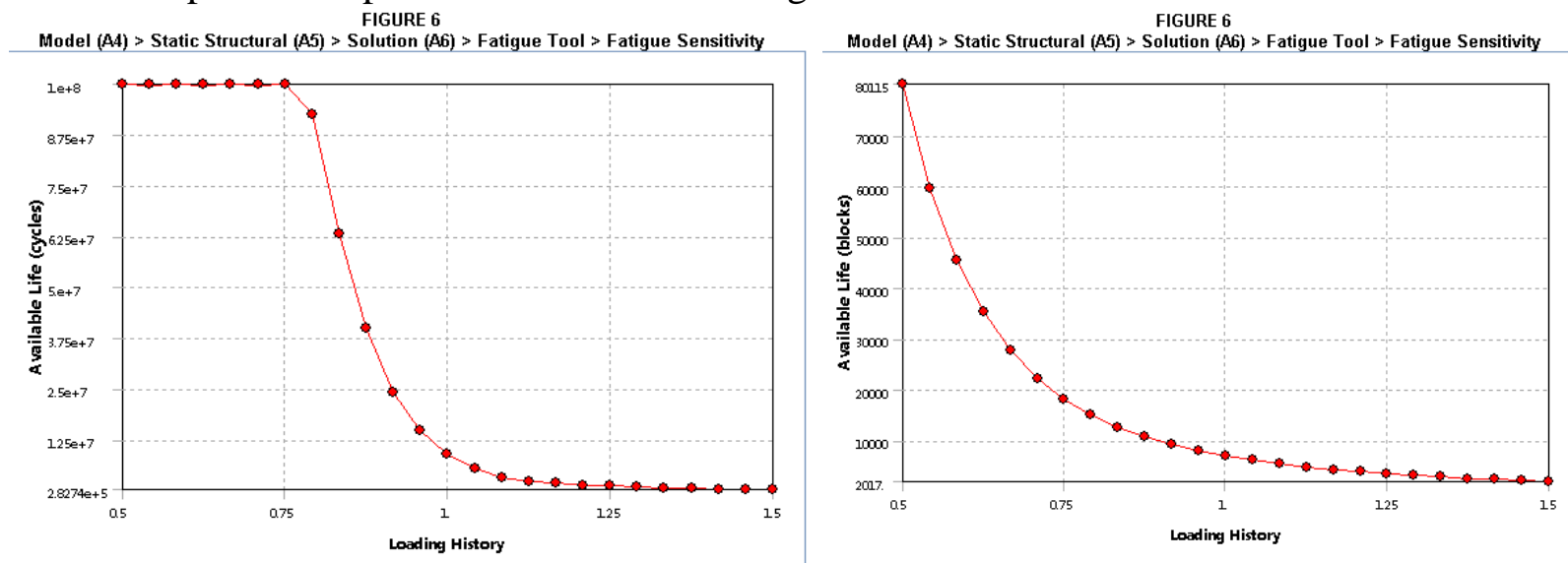

Figure 6: Load - time Graph

By fatigue analysis, get the derived load - time curve (Figure 6), in the case of the load $9.35 \times 10^{7} \mathrm{~Pa}$, the curve plunged; and under a load of $1 \times 10^{8} \mathrm{~Pa}$ square cross-section can also work for some time, from the point of fatigue resistance, square cross-section is also superior.

(2) Due to the special environmental, primary material is alloy GH2036, it resistant high temperature, yield strength $680 \mathrm{MPa}$, according to the safety factor of 1.2 to calculate the maximum allowable stress is $340 \mathrm{MPa}$, and according to the Workbench analysis results, steel material weight is relatively large, which greatly increases the stress of mechanical mechanism. Aluminum alloy and structural steel static stress analysis and fatigue life analysis and comparison:FIG.7 is about the comparison of stress load when applied on both cylinder fully extended, the max stress of steel up to $2.03 \times 10^{8} \mathrm{~Pa}$, and the stress on aluminum is $2.31 \mathrm{MPa}$, the tensile yield strength and compressive yield strength of aluminum was $280 \mathrm{MPa}$; observing the maximum strain of the two materials (Fig. 8), it is easy to obtain the maximum strain of the steel material $5.25 \times 10^{-3} \mathrm{~m} / \mathrm{m}$, and aluminum up to $5.94 \times 10^{-3} \mathrm{~m} / \mathrm{m}$, so the total value on displacement (FIG. 9), aluminum is relatively large, it is $0.78 \mathrm{~m}$, and steel is only $0.64 \mathrm{~m}$, both are less than the tolerance $1 \mathrm{~m}$. Based on the analysis of static stress, analyzing the load - time curve (Fig. 10) we can see with the change of loading weight, the using time of aluminum alloy will be greatly changed.

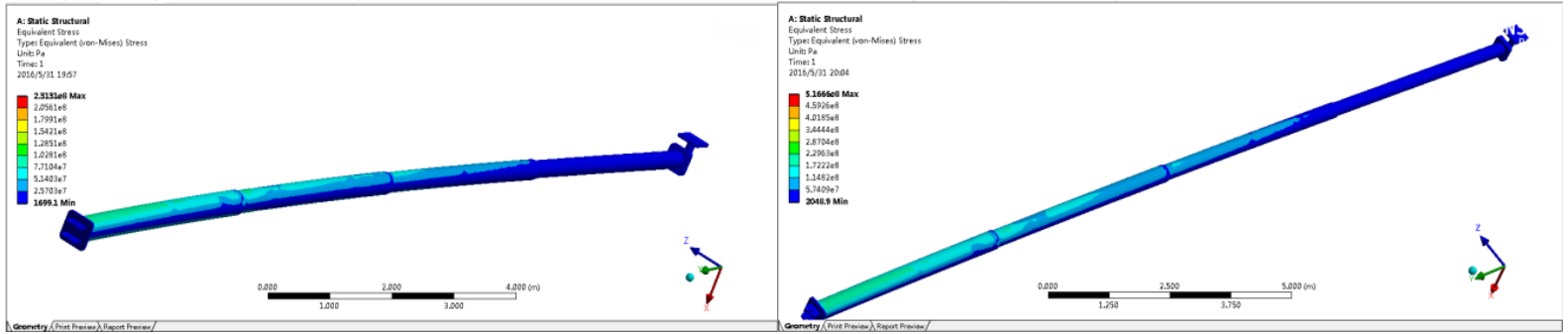

Figure 7: Stress Comparison 


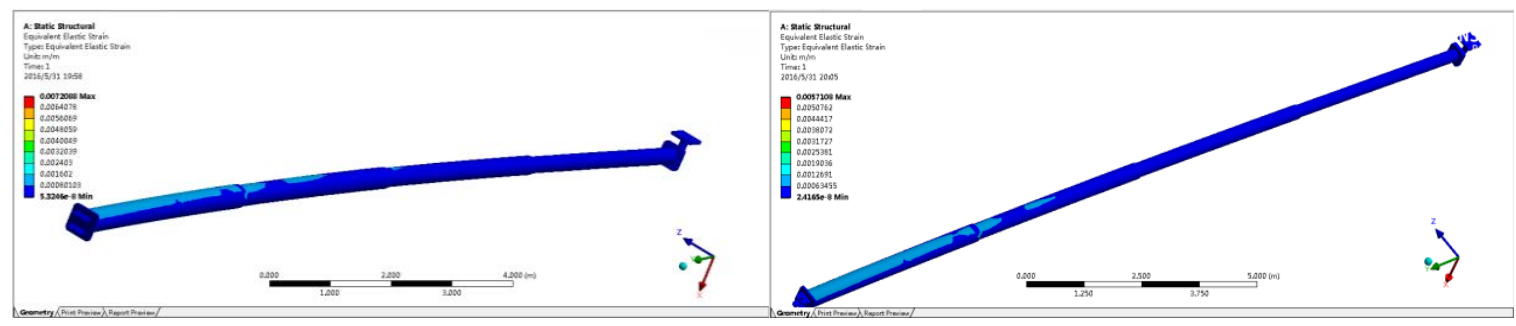

Figure 8: Strain Comparison

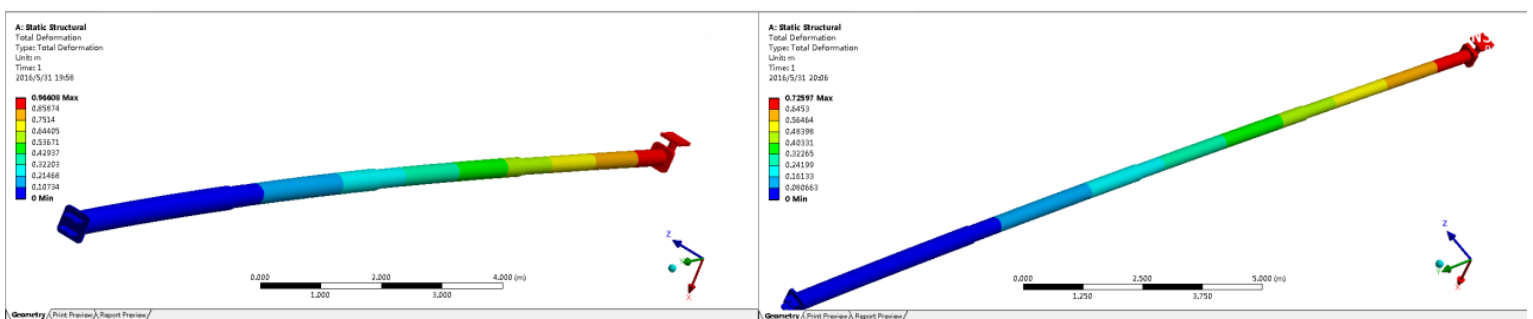

Figure 9: Comparison of total displacement
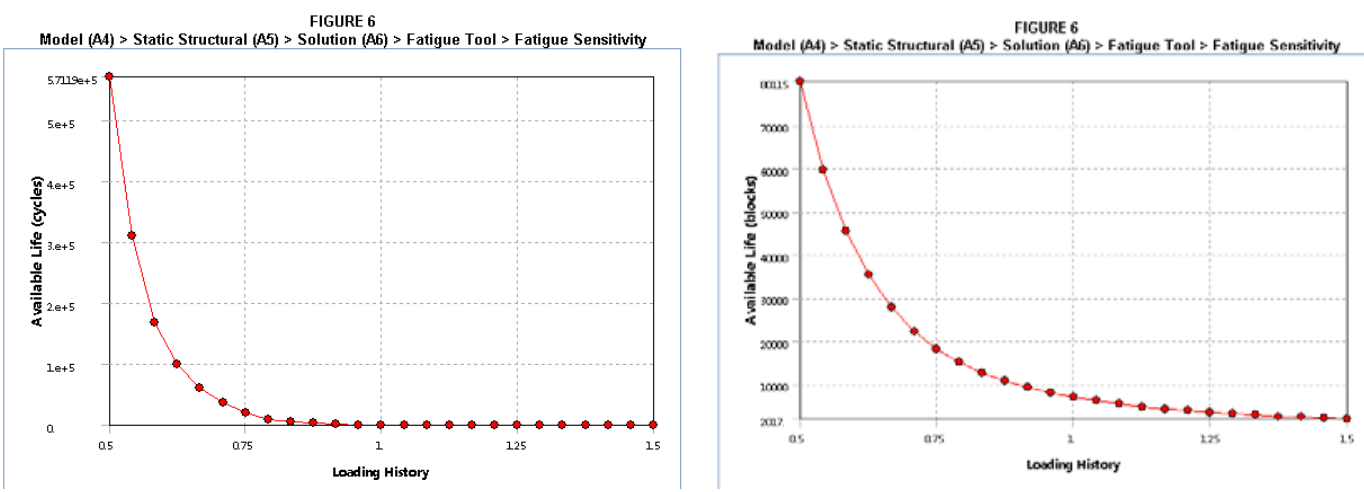

Figure 10: Life Analysis

According to the above results, aluminum is lighter than steel, but in terms of the stiffness, aluminum is not as good as steel; melting point of aluminum is $550^{\circ} \mathrm{C} \sim 630^{\circ} \mathrm{C}$, and steel is above $1300^{\circ} \mathrm{C}$, steel is more fire-adapted. From the point of life-span, steel superior to aluminum, because of its greater stiffness, small deformation; it can be adapted to the total displacement distance cantilever model requirements. When selecting material, priority to the safety of use, steel is more suitable.

\section{Structural analysis performed for the cylinder 1}

(1) Static Analysis

Since the first-stage telescopic cylinder withstand the maximum load, define the bottom of model the rigid constraints, apply internal pressure of $2000 \mathrm{~Pa}$, under the conditions of imposed $1500 \mathrm{~N}$ enable gravity load on the cylinder end, get the static analysis cloud figure 11 . Stress can be seen up to $38.46 \mathrm{MPa}$; the cylinder head stress suffered is minimal, only $10.56 \mathrm{MPa}$.

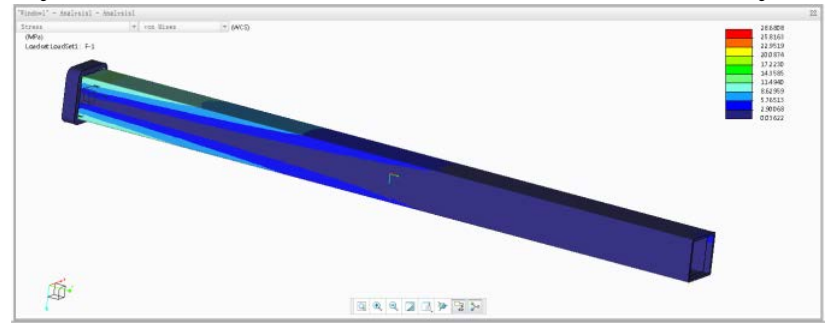

Figure 11: Static Analysis cloud

(2) Sensitivity analysis 
From the global sensitivity analysis (FIG12, 13) we can see that with the increase in the axial direction on the model size, load changes linearly, similar changes also occur in the curve of stress and strain.



Figure 12:Global sensitivity analysis cloud

Figure 13: Global sensitivity analysis graph

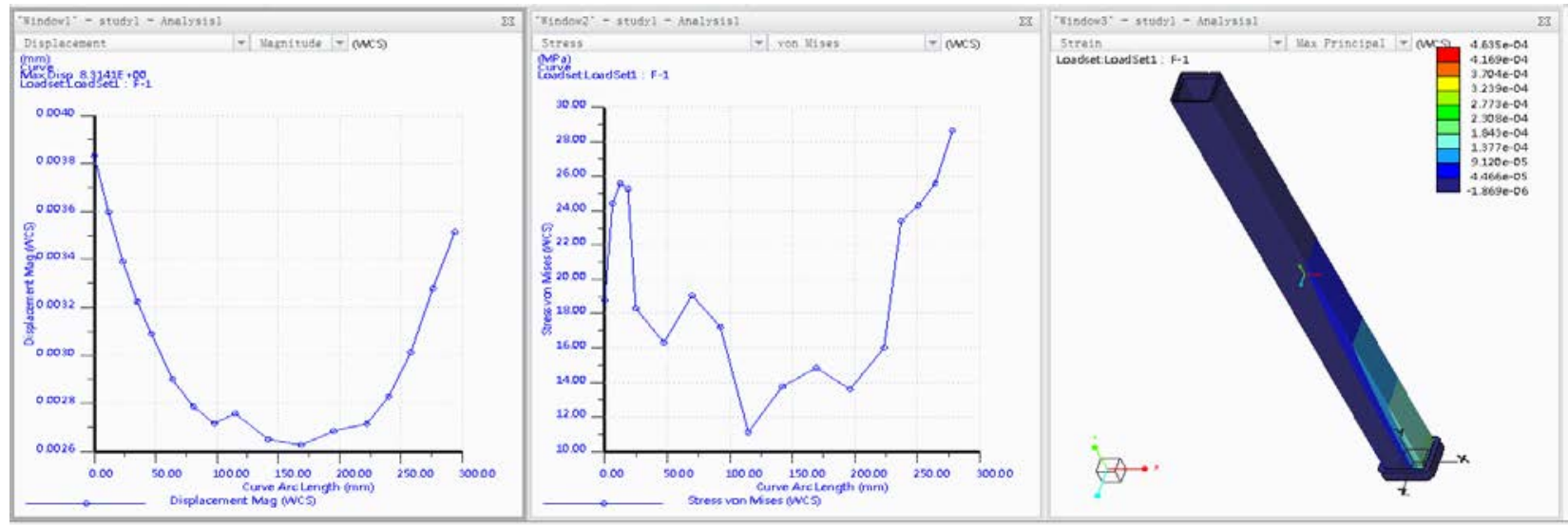

Figure 14: Local Sensitivity Analysis

The inner cross-sectional width affect the local sensitivity analysis as shown in FIG. 14, the total displacement, stress and strain with width variation can be found is not linear, it is possible to optimize the design for this data.

Optimization based on Creo3.0 Simulate is by setting optimization goal, optimization conditions and parameters range etc., using secondary optimization arithmetic to optimize the structure of the cylinder. Based on the results above to optimize the length and cross-sectional dimensions to meet the rigidity strength conditions, minimize the overall weight of the cylinder. Optimization results are as follows:

Target: Minimize: Total mass

Restrictions: Analysis1 (static analysis) Load Collection: LoadSet1 (preload)

max_stress_vm $<3.8 \times 10^{2}$ (Yield stress limit)

Optimization convergence tolerance: $2 \%$

The maximum number of iterations: 20 times

Optimization of design parameters: d27 4895.6; d35 246.2

Target: $0.361 \mathrm{e}+002$ (Unit: Kg)

\section{Conclusion}

(1) Based on ANSYS Workbench analysis of circular cross section and square cross section, compare static analysis and fatigue analysis, we can see square cross section is more suitable for the design of pneumatic telescopic arm. Due to the stiffness of steel is large, adapt to fire environment, steel is more suitable.

(2) Based on the analysis and optimization of Creo3.0 simulate environment, mainly analyzed and optimized the forces of the cylinder 1 and reduced the weight of the overall structure, under the 
condition that the cylinder material failure does not occur, finally the cylinder was reduced from the original 396kg to $361 \mathrm{~kg}$.

\section{Acknowledgement}

This research was financially supported by the Hebei Province Science and Technology Foundation (No.Z2015072, No.15275508) and NCIST Foundation (No. 3142015023).

\section{References}

[1] X. M. Li, B. Z. Ren, X. H. Liu, Urban high-rise building fire safety and fire escape Strategy [J]. China Safety Science .2009 (08)

[2] L. L. Dong, Y. P. Zhao, L. Q. Liang, Y. Zhu, G. H. Duan. Summary of Mechanical Optimization Design Method [J].Machine Tool \& Hydraulics .2010 (08)

[3] W. C. Zhou, X. T. Liu, Y. Xing, L. Lin. FEM analysis of telescopic aerial work platform [J]. 2011 Construction machinery (07)

[4] Y. L. Han, X. L. Wen, B. L. Wang. Based on ANSYS finite element analysis and optimization of hydraulic cylinder [J].Mine Machinery .2011 (09)

[5] A. M. Ji, Y. L. Luo. Crane telescopic boom section optimization design [J]. Construction Mechanization. 2006 (03)

[6] H. Liu .ANSYS15.0 finite element analysis from entry to the master [M] Beijing: Mechanical Industry Press, 2014: 327-364.

[7] Y. Y. Wang, L. Zhu, Materials for Mechanical Engineering [M] Beijing: Mechanical Industry Press, 2008.12 